\title{
MELTING OF POLYMER BLENDS IN SINGLE-SCREW EXTRUSION - AN EXPERIMENTAL STUDY
}

\author{
S. M. Cunha*, A. Gaspar-Cunha, J. A. Covas \\ Department of Polymer Engineering, IPC/I3N \\ University of Minho - Portugal
}

\begin{abstract}
Melting is a major step in plasticating single screw extrusion, but most of the existing phenomenological know how was gathered by performing Maddock-type experiments with homopolymers. Given the current widespread industrial use of polymer blends, it is worth determining whether the same mechanisms and mathematical models apply, or whether different sequences develop. This work reports the results of Maddock-type experiments using a PA6/PP blend, both in its immiscible and compatibilized varieties. A melting mechanism combining the features of the classical Tadmor mechanism and of the dispersed melting mechanism, also previously reported in the literature, was observed.
\end{abstract}

KEYWORDS: Melting, Melting mechanism, Polymer blends, Single-screw extrusion.

\section{INTRODUCTION}

Melting is a major step in plasticating single screw extrusion, as it influences important global process characteristics, such as output and degree of mixing. Experimental melting studies were initiated by Maddock in the fifties, yielding a qualitative phenomenological understanding of the underlying mechanisms [1]. Based on this evidence, Tadmor developed the corresponding mathematical description [2], which in fact formed the basis of subsequent more sophisticated analyses [3-9]. In his model, Tadmor assumes that the solid pellets are compacted and form a continuous bed, which is separated from the inner barrel wall by a thin melt film. The relative velocity between solids and barrel pushes this film into a melt pool, which forms adjacent to the pushing flight. In turn, the melt pool pushes the solid bed against the trailing flight, exposing more solids for melting at the solids-melt film interface.

In contrast with the Maddock/Tadmor mechanism, melting of individual solid pellets suspended in the melt has been experimentally observed when using specific screw geometries [10-12], Rauwendaal having put forward the first mathematical approach of this dispersed melting sequence [13]. Peng [14] and Potente and Pape [15] suggested improvements to this model.

The above experimental observations and models typically consider processing of homopolymers, or of very simple polymer systems. The study of melting of (immiscible or reactive) polymer blends is much less common and seems to be limited to twin screw extrusion [16-18]. It is well known that the morphologies formed upon melting will influence the final morphologies obtained which, in turn, will determine the mechanical properties [19-21].

Since single-screw extruders are nowadays often used to manufacture extrudates from complex polymer systems, it is interesting to revisit the melting stage, i.e., it is important to use Maddock-type experiments to understand whether the melting behaviour of systems such as polymer blends is different that of conventional models. If that is the case, then it is also important to develop the corresponding mathematical descriptions.

This work reports experimental observations of melting of PA6/PP blends in their physical and compatibilized forms.

\section{EXPERIMENTAL}

\subsection{MATERIALS}

Polyamide 6 / Polypropylene (physical blends, PA6/PP and compatibilized in situ with Maleic anhydride grafted Polypropylene, PA6/PP/PP-g-MA) were selected for this study, the specific grades being presented in Table 1 (they were chosen so that the viscosity ratio of the physical blend would be approximately 1). DSC thermograms showed that the melting temperature of PP and PA6 was $166^{\circ} \mathrm{C}$ and $226^{\circ} \mathrm{C}$, respectively.

Table 1: Materials selected

\begin{tabular}{|c|c|c|}
\hline Material & Trade name & Supplier \\
\hline PP & ISPLEN 030 G1E & REPSOL \\
\hline PP-g-MA & OREVAC CA 100 & ARKEMA \\
\hline PA6 & Akulon F 130 & DSM \\
\hline
\end{tabular}

\footnotetext{
* Corresponding author: University of Minho - Department of polymer engineering - Campus de Azurém 4800-058 Guimarães, phone: +351253510337, fax: +351253510339, e-mail: denebe@dep.uminho.pt
} 


\subsection{EQUIPMENT}

The blends were prepared in a prototype modular single screw extruder, with a screw diameter of $30 \mathrm{~mm}$ and L/D $=30$, fitted with material sampling devices and pressure transducers along the barrel (see [22] for details), and with a mechanical system for fast screw extraction. The screw profile can be modified using the same modularity approach as that of co-rotating twin screw extruders.

\subsection{EXPERIMENTAL PROCEDURE}

Many Maddock-type experiments were performed in order to investigate the effect on melting of changes in blend composition, screw speed, barrel temperature profile and screw geometry.

In each case, the polymers were pre-mixed in a drum mixer for 10 minutes before extrusion. After reaching extrusion steady state (typically 20 minutes), screw rotation was interrupted, the die was quickly removed and the screw was extracted from the barrel by means of the available mechanical contraption, the whole operation taking approximately 1 minute. The polymer helix in the screw channel was further cooled with compressed air and removed for subsequent analysis.

Cross-sections were cut from polymer helices at regular down-channel intervals and immersed in epoxy for $24 \mathrm{~h}$ before polishing. Later, the samples were submitted for one hour to a dyeing bath at $80^{\circ} \mathrm{C}$ of an aqueous solution of the reactive LANASOL Blue dye, in order to tint PA6 and thus distinguish between PA6 and PP. The dye's $\alpha$ bromoacrylamido group can bond covalently with the amine groups of PA6 [23], all the surface of PA6 becoming blue. The samples were observed using a Olympus stereoscopic magnifying glass and photographed with a Leica digital camera, the images being subsequently analysed with the Image-Pro Plus 4.5 software.

\section{EXAMPLE OF RESULTS}

Figure 1 shows representative cross-sections obtained from the polymer helices removed from the screw, after screw extraction. The cross-sections on top refer to the immiscible PA6/PP (80/20) blend, whereas those at the bottom correspond to the compatibilized PA6/PP/PP-gMA (50/40.6/9.4) counterpart. In the first screw turns the cross-sections are not complete, as during screw extraction loose pellets detached and were lost. PA6 is seen either as blue or green, depending on the photos's contrast or brightness. Anyway, it is clearly distinguishable from PP. Moreover, solids and molten material are also easily differentiated.

Observation of the series of cross-sections on each photo reveals that the melting mechanism of the PA6/PP system is much more complex than the traditional sequence reported in literature for homopolymers. It seems to combine elements of Tadmor's and dispersed mechanisms, hence it will be denoted as "hybrid melting mechanism".
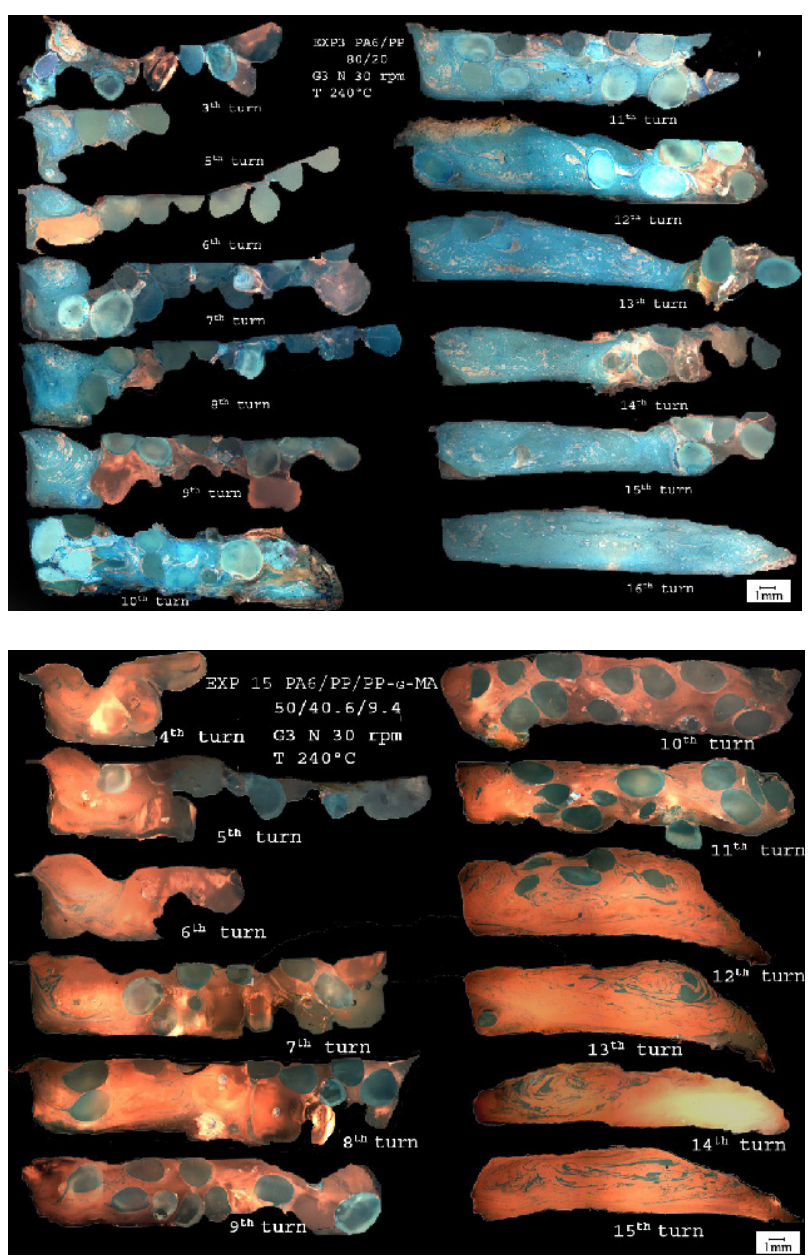

Figure 1: Cross-sections at various screw turns for two different experiments. Top: PA6/PP (80/20); bottom: PA6/PP/PP-g-MA (50/40.6/9.4)

Usually, the representation of the evolution of melting along the screw channel is made in terms of a reduced solids width, $\mathrm{X} / \mathrm{W}$ (local solids width to channel width ratio), as the solid bed is assumed as approximately rectangular. Figure 2 contains not only this type of information, but shows also the evolution of melting in terms of the relative area of solids (As/At where As is the total cross-sectional area occupied by solids and At is the total area of the cross-section) and number of solid particles suspended in the melt. The data refers to PA6/PP physical blends with different compositions $(80 / 20,50 / 50$ and $20 / 80)$, which were processed under the same operating conditions $(220 / 230 / 240 / 240 / 240 /$ $240^{\circ} \mathrm{C}$ from hopper to die, screw speed of $30 \mathrm{rpm}$ ) and identical screw geometry (compression ratio $=2.5$, feeding compression and metering zones $10 \mathrm{D}$ long). This set temperature profile resulted from the need to deal with processing two blend components with quite distinct melting temperatures. The resulting heat conduction, together with the small size of the extruder, promoted early melting of both materials. Also, melting was completed well before the end of the compression zone. 
The evolution of the reduced solids width (X/W) indicates that melting is completed earlier in the screw for blends richer in PP. Differences between the axial profiles of $\mathrm{X} / \mathrm{W}$ and As/At, specially for the blends richer in PP, are a sign that either the solid bed height no longer remains essentially constant, or that changes in the classical melting mechanism are taking place. This is confirmed by the plot of the evolution of the number of suspended solid particles, which is an evidence of the development of a parallel mechanism. This number is higher for the 50/50 blend, and particles appear earlier when the quantity of PP in the blend increases.

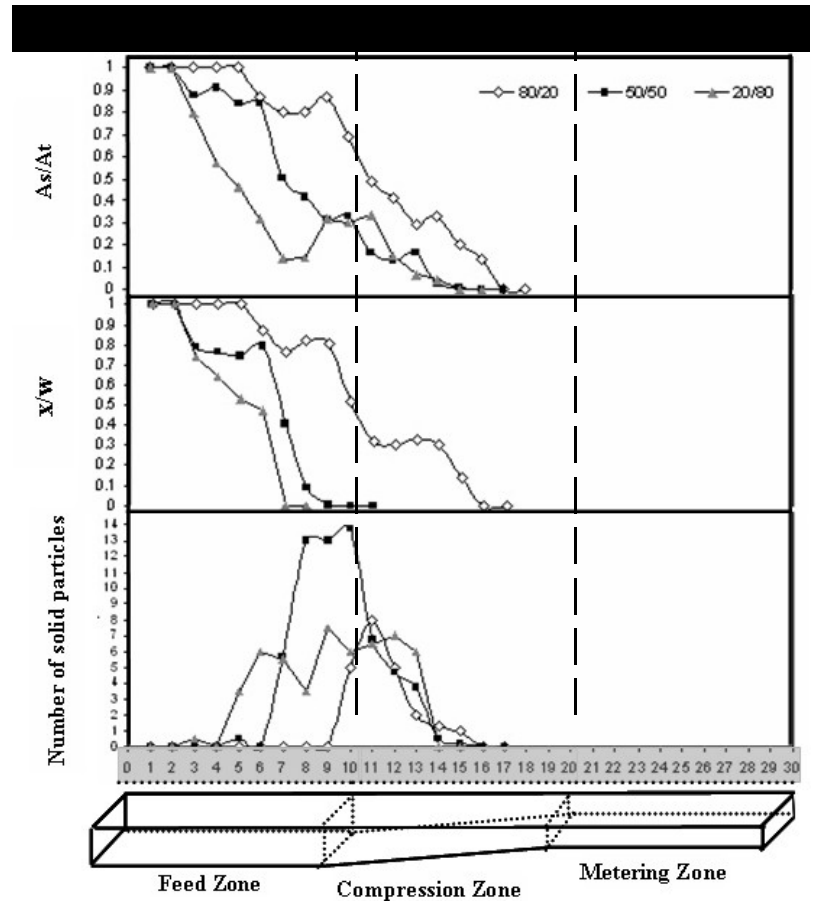

Figure 2: Evolution of melting along the screw in terms of reduced solids width, relative area occupied by solids and number of solid particles suspended in the melt (top to bottom)

Figure 2 provides information at the larger scale of the presence of solid versus molten material. The next scale of analysis implies discrimination between PA6 and PP. This is done in Figure 3, where the contribution of each material to the melting progress of the blends is shown. Each plot refers to one of the compositions and represents the area of each molten blend component, as well as that of the blend, normalized to the area of the channel cross-section. The horizontal lines correspond to the values expected for each polymer after complete melting, taking into account their relative density and relative percentage.

In the feeding zone (screw turns 1-10), the melting rate of the blends is essentially determined by the PP melting rate, whereas in the compression zone (screw turns 1120) the role of PA6 seems predominant. Moreover, in the feed zone (screw turns 1-10), the higher the PP content in the blend, the higher the melting rate of this polymer.
Conversely, the melting rate of PA6 is practically the same, but increases drastically in the compression zone.
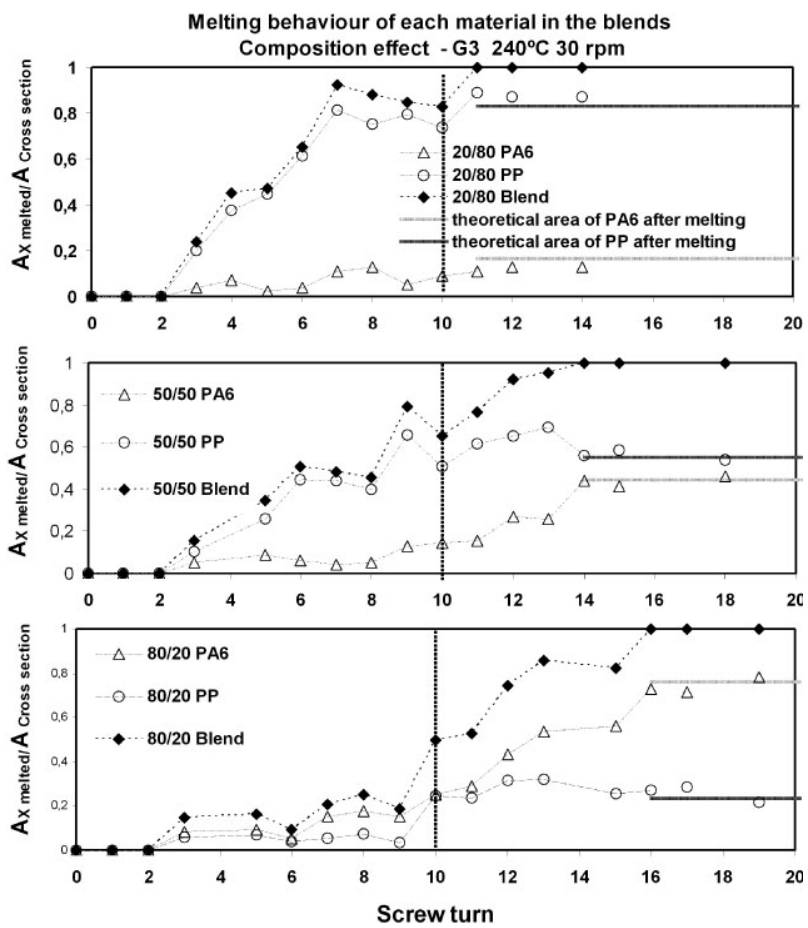

Figure 3: Contribution of each blend component to the melting evolution of the blend

Figure 3 shows also that despite of the differences in the melting temperature of the two blend components (circa $60^{\circ} \mathrm{C}$ ) both materials start melting at approximately the same location, even if at different rates. In fact, for the PA6/PP 80/20 blend some PP pellets are visible at crosssections that are essentially molten. Melting of each pellet will depend on its location in the channel. For example, pellets contacting the barrel surface will melt first, and more or less simultaneously regardless of the polymer they are made of, due to the significant contribution of heat dissipated by friction.

It is worth noting that in laboratory extruders the size of individual pellets is relevant, particularly in terms of the screw channel depth, which may hinder scale up of the results to industrial production machines. However, Maddock-type experiments are easier to perform in smaller machines.

\section{CONCLUSIONS}

A number of Maddock-type experiments performed with immiscible and compatibilized polymer blends, under a variety of operating conditions and screw profiles, showed that the melting sequence in conventional screw extruders follows a more complex mechanism than that reported for homopolymers, and described mathematically by Tadmor. Instead, a competition between Tadmor's and dispersed mechanisms is observed, i.e., an hybrid mechanism develops. For a 
complete understanding of the underlying physical phenomena, future work will concentrate on a finer observation scale, i.e., on monitoring the evolution of morphology upon melting.

\section{ACKNOWLEDGEMENTS}

The authors are grateful to Portuguese Fundação para a Ciência e Tecnologia for supporting this work under grant SFRH/BD/19997/2004 and to DSM, the Netherlands, for supplying PA6.

\section{REFERENCES}

[1] B. H. Maddock. SPE Journal, 15:383-389, 1959.

[2] Z. Tadmor, D.I. Marshall, I. Klein. Fundamentals of plasticating extrusion. I. A theoretical model for melting. Polym. Eng. Sci., 6:185-190, 1966.

[3] R. C. Donovan. A theoretical melting model for plasticating extruders. Polym. Eng. Sci., 11:247-257, 1971.

[4] I. R. Edmondson, R.T. Fenner. Melting of thermoplastics in single screw extruders. Polymer, 16:49-56, 1975.

[5] J. T. Lindt. A dynamic melting model for a singlescrew extruder. Polym. Eng. Sci., 16: 284-291, 1976.

[6] J. Shapiro, A. L. Halmos, J. R. A. Pearson. Melting in single screw extruders. Polymer, 17:905-918, 1976.

[7] J. T. Lindt, B. Elbirli. Effect of the cross-channel flow on the melting performance of a single-screw extruder. Polym. Eng. Sci., 25: 412-418, 1985.

[8] C. Rauwendaal. An improved analytical melting theory. Adv. Polym. Tech., 9: 331-336, 1989.

[9] C. D. Han, K.Y. Lee, N. C. Wheeler. A study on the performance of barrier-screw extruders. Polym. Eng. Sci., 31:831-841, 1991.

[10]F. Zhu, L. Chen. Studies on the theory of single screw plasticating extrusion. Part I: A new experimental method to extrusion. Polym. Eng. Sci., 31:1113-1116, 1991.

[11] R. J. Nichols, F. Kheradi. Modern plastics, 12:70, 1984.

[12] S. Jakopin, P. Franz. AIEChE Diamond Jubilee Meeting tech. papers, XXXVI: 1984.

[13] C. Rauwendaal. Dispersed solids melting theory. SPE ANTEC Tech. papers : 2232-2237, 1993.

[14]H. X. Huang, Y. C. Peng. Theoretical modeling of dispersive melting mechanism of Polymers in an Extruder. Adv. Polym. tech. papers, 4: 343-352, 1993.

[15]H. Potente and J. Pape. The melting behaviour of solid particles surrounded by melt under shear conditions. In $17^{\text {th }}$ Annual Meeting of the PPS, 2001.

[16] H. Potente, S. Krawinkel, M.Bastian, M. Stephan, P. Pötschke. Investigation of the melting behaviour and morphology of polymer blends in the melting zone of twin-screw extruders. Journ. of Appl. Polym. Sci., 8: 1986-2002, 2001.

[17]H. Chen, U. Sundararaj, K. Nandakumar. Investigation of melting mechanism in a twin-screw extruder using a pulse method and on-line measurement. SPE ANTEC Tech. papers: 122-127, 2004.

[18] B. Vergnes, F. Berzin. Modeling of reactive systems in twin-screw extrusion: challenges and applications. C. R. Chimie, 9: 1409-1418, 2006.

[19] U. Sundararaj, C. W. Macosko, R. J. Rolando, H. T. Chan. $\mathrm{T}$ Morphology Development in polymer blends. Polym. Eng. Sci., 32: 1814 -1823, 1992.

[20] A. V. Machado, J. A. Covas, M. Duin. A study of grafting reactions during processing of polyolefins. Adv. Polym. Tech., 23: 196-210, 2004.

[21] A. V. Machado, V. Yquel, J. A. Covas, J. Flat, N. Ghamri, A. Wollny. The effect of compatibilization routr of PA/PO blends on the physico-chemical phenomena developing along a twin-screw extruder. Macromo. Symp., 233: 86-94, 2006.

[22] A. V. Machado, J. A. Covas, M. Duin. Evolution of morphology and of chemical conversion along the screw in a corotating twin-screw extruder. J. Appl. Polym. Sci., 71: 135-141, 1999.

[23] J. S. Church, A. S. Davie, P. J. Scammells, D. J. Tucker. Lanasol dyes and wool fibres. Part I: Model studies on the mechanism of dye fixation in a mixed solvent system. Dyes and Pigments, 39: 291-312, 1998. 